\title{
Test beam evaluation of silicon strip modules for ATLAS phase-II strip tracker upgrade
}
A.J. Blue ${ }^{1, *}$, A.A. Affolder ${ }^{22}$, X. Ai ${ }^{13,25}$, P.P. Allport ${ }^{3}$, J.-H. Arling ${ }^{9}$, R.J. Atkin ${ }^{5}$, L.S. Bruni ${ }^{2}$, I. Carli ${ }^{18}$, G. Casse ${ }^{16}$, L. Chen ${ }^{13,25,29}$, A. Chisholm ${ }^{7}$, K. Cormier ${ }^{26}$, W. Cunningham ${ }^{1}$, P. Dervan ${ }^{16}$, S. Diez ${ }^{9}$, Z. Dolezal ${ }^{18}$, J. Dopke ${ }^{21}$, E. Dreyer ${ }^{24}$, J. Dreyling-Eschweiler ${ }^{9}$, C. Escobar ${ }^{12}$, V. Fabiani ${ }^{17}$, V. Fadeyev ${ }^{22}$, J. Fernandez-Tejero ${ }^{8}$, C. Fleta ${ }^{8}$, A. Gabrielli ${ }^{15}$, B. Gallop ${ }^{21}$, C. García Argos ${ }^{10}$, A. Greenall ${ }^{16}$, I.M. Gregor ${ }^{9}$, G. Greig ${ }^{24}$, F. Guescini ${ }^{27}$, K. Hara ${ }^{28}$, M. Hauser ${ }^{10}$, Y. Huang ${ }^{13,25}$, R.F.H. Hunter ${ }^{6}$, J.S. Keller ${ }^{6}$, C.T. Klein ${ }^{4}$, P. Kodys ${ }^{18}$, U. Koetz ${ }^{9}$, T. Koffas ${ }^{6}$, Z. Kotek ${ }^{19}$, J. Kroll ${ }^{19}$, S. Kühn ${ }^{7,10}$, S.J. Lee ${ }^{6}$, Y. Liu ${ }^{13,25}$, K. Lohwasser ${ }^{23}$, L. Mészárosová ${ }^{18}$, M. Mikestikova ${ }^{19}$, M. Miñano Moya ${ }^{12}$, R. Mori $^{10}$, B. Moser ${ }^{10}$, K. Nikolopoulos ${ }^{3}$, R. Peschke ${ }^{9}$, G. Pezzullo ${ }^{7}$, P.W. Phillips ${ }^{21}$, L. Poley ${ }^{9}$, M. Queitsch-Maitland ${ }^{9}$, F. Ravotti ${ }^{7}$, D. Rodriguez ${ }^{12}$, E. Rossi ${ }^{9}$, A. Rummler ${ }^{7}$, C. Sawyer ${ }^{21}$, D. Sperlich ${ }^{11}$, S. Sullivan ${ }^{21}$, J. Suzuki ${ }^{28}$, M. Sykora ${ }^{18}$, E. Tahirovic ${ }^{20}$, O. Theiner ${ }^{18}$, J. Thomas ${ }^{3}$, Y. Unno ${ }^{14}$, S. Wada ${ }^{28}$, M. Warren ${ }^{30}$, M. Wiehe ${ }^{10}$, S. Wonsak ${ }^{16}$, M. Wormald ${ }^{16}$, K. Wraight ${ }^{1}$, N. Zakharchuk ${ }^{9}$, H. Zhu ${ }^{13,25}$

\footnotetext{
${ }^{1}$ SUPA School of Physics and Astronomy, University of Glasgow, Kelvin Building, University Avenue, Glasgow G12 8QQ, Scotland, United Kingdom

${ }^{2}$ University of Amsterdam/NIKHEF, Amsterdam, Netherlands

${ }^{3}$ Particle Physics Group, School of Physics and Astronomy, University of Birmingham, Birmingham B15 2TT, United Kingdom

${ }^{4}$ Cavendish Laboratory, University of Cambridge, JJ Thomson Avenue, Cambridge CB3 OHE, United Kingdom

${ }^{5}$ Department of Physics, University of Cape Town, University Avenue, Rondebosch, Cape Town 7700, South Africa

${ }^{6}$ Physics Department, Carleton University, 1125 Colonel By Drive, Ottawa, Ontario, K1S 5B6, Canada

${ }^{7}$ CERN, Geneva, Switzerland

${ }^{8}$ Centro Nacional de Microelectronica (IMB-CNM, CSIC), Campus UAB-Bellaterra, 08193 Barcelona, Spain

${ }^{9}$ Deutsches Elektronen-Synchrotron, Notkestraße 85, 22607 Hamburg, Germany

${ }^{10}$ Physikalisches Institut, Albert-Ludwigs-Universität Freiburg, Hermann-Herder-Str. 3, 79104 Freiburg-im-Breisgau, Germany

${ }^{11}$ Institut für Physik, Humboldt-Universität zu Berlin, Newtonstraße, Berlin, Germany

${ }^{12}$ Instituto de Física Corpuscular (IFIC) - CSIC-University of Valencia, Parque Científico, C/Catedrático José Beltrán 2, E-46980 Paterna, Spain

${ }^{13}$ Institute of High Energy Physics, Chinese Academy of Science, Beijing, China

${ }^{14}$ IPNS, KEK, 1-1 Oho, Tsukuba, Ibaraki 305-0801, Japan

${ }^{15}$ Physics Division, Lawrence Berkeley National Laboratory, Berkeley, CA, USA

${ }^{16}$ Particle Physics, University of Liverpool, Oliver Lodge Building, Department of Physics, Oxford Street, Liverpool, L69 7ZE, United Kingdom

${ }^{17}$ Institute for Mathematics, Astrophysics, and Particle Physics, Radboud University Nijmegen/NIKHEF, Nijmegen, Netherlands

${ }^{18}$ Faculty of Mathematics and Physics, Charles University, V Holesovickach 2, Prague, CZ18000, Czech Republic

${ }^{19}$ Academy of Sciences of the Czech Republic, Institute of Physics, Na Slovance 2, 18221 Prague 8, Czech Republic

${ }^{20}$ School of Physics and Astronomy, Queen Mary University of London, London, United Kingdom

${ }^{21}$ Particle Physics Department, STFC Rutherford Appleton Laboratory, Chilton, Didcot, OX11 OQX, United Kingdom

${ }^{22}$ Santa Cruz Institute for Particle Physics (SCIPP), University of California, Santa Cruz, CA 95064, USA

${ }^{23}$ Department of Physics and Astronomy, University of Sheffield, Hicks Bldg, Sheffield S3 7R, United Kingdom

${ }^{24}$ Department of Physics, Simon Fraser University, Burnaby, British Columbia, Canada

${ }^{25}$ State Key Laboratory of Particle Detection and Electronics, Beijing, China

${ }^{26}$ Department of Physics, University of Toronto, 60 Saint George St., Toronto M5S 1A7, Ontario, Canada

${ }^{27}$ Physical Sciences Division, TRIUMF, 4004 Wesbrook Mall, Vancouver, BC, Canada V6T 2A3

${ }^{28}$ Institute of Pure and Applied Sciences, University of Tsukuba, 1-1-1 Tennodai, Tsukuba, Ibaraki 305-8571, Japan

${ }^{29}$ University of Chinese Academy of Sciences, Beijing, China

${ }^{30}$ Department of Physics and Astronomy, University College London, London, United Kingdom
}

\footnotetext{
* Corresponding author.

E-mail address: andrew.blue@glasgow.ac.uk (A.J. Blue).
} 


\section{A R T I C L E I N F O}

\section{Keywords:}

Semiconductor

Silicon

Detector

\section{A B S T R A C T}

The planned High Luminosity Large Hadron Collider is being designed to maximise the physics potential of the LHC with 10 years of operation at instantaneous luminosities of $7.5 \times 10^{34} \mathrm{~cm}^{-2} \mathrm{~s}^{-1}$. A consequence of this increased luminosity is the expected radiation damage requiring the tracking detectors to withstand hadron fluence to over $1 \times 10^{15} 1 \mathrm{MeV}$ neutron equivalent per $\mathrm{cm}^{2}$ in the ATLAS Strips system. Fast readout electronics, deploying $130 \mathrm{~nm}$ CMOS front-end electronics are glued on top of a silicon sensor to make a module. The radiation hard n-in-p micro-strip sensors used have been developed by the ATLAS ITk Strip Sensor collaboration and produced by Hamamatsu Photonics. A series of tests were performed at the DESY-II test beam facility to investigate the detailed performance of a strip module with both $2.5 \mathrm{~cm}$ and $5 \mathrm{~cm}$ length strips before irradiation. The DURANTA telescope was used to obtain a pointing resolution of $2 \mu \mathrm{m}$, with an additional pixel layer installed to improve timing resolution to $\sim 25 \mathrm{~ns}$. Results show that prior to irradiation a wide range of thresholds (0.5-2.0 fC) meet the requirements of a noise occupancy less than $1 \times 10^{-3}$ and a hit efficiency greater than $99 \%$.

\section{Introduction}

\subsection{ATLAS upgrade for HL-LHC}

The High Luminosity Large Hadron Collider (HL-LHC) will operate at an ultimate peak instantaneous luminosity of $7.5 \times 10^{34} \mathrm{~cm}^{-2} \mathrm{~s}^{-1}$ which corresponds to approximately 200 inelastic proton-proton collisions per beam crossing (pile-up) [1]. It will be operational for more than 10 years and in that time ATLAS aims for a total data set of $4000 \mathrm{fb}^{-1}$. To operate at the higher data rates, withstand the radiation levels, and maintain low occupancy in the high pile-up environment, the current ATLAS Inner Detector (ID) [2] will be replaced by a new Inner Tracker (ITk). The ITk will be an all-silicon tracking system that consists of a pixel detector at small radius close to the beam line and a large area strip tracker surrounding it.

\subsection{ITK strips system}

The ITk strips system is composed of modules [3]. The strip modules are single-sided with the hybrid circuits carrying the front-end microelectronic ASICs glued to the sensor surface. Modules are sandwiched on both sides of low mass carbon-fibre support structures with embedded bi-phase $\mathrm{CO}_{2}$ cooling. Each module consists of multiple rows of strips with a pitch of $74.5 \mu \mathrm{m}$ in the central (barrel) region, and ranging from $69 \mu \mathrm{m}$ to $85 \mu \mathrm{m}$ in the forward (end-cap) regions. The local support structures for the barrels are staves with 14 modules on each side while the end-cap discs are built from petals with 9 modules of different types per side. For the central barrel region of the strips system, the strips on the inner two cylinders are $24.1 \mathrm{~mm}$ long (short-strips) and those on the outer two cylinders are $48.2 \mathrm{~mm}$ long (long-strips). The short-strip barrel modules contain two hybrids, each with ten ABC read-out ASICs, whereas the long-strip modules contain one hybrid with ten ABC ASICs.

\subsection{Motivation for testing}

It is important to understand the performance of the strip modules before irradiation, with the most critical parameters being the Equivalent Noise Charge (ENC) in electrons, the gain, the collected charge, the hit efficiency, and the noise occupancy. In addition to standard lab tests, the characterisation of a module operated at a particle test beam is then a vital tool for the evaluation of the module and its associated components.

\section{Setup}

\subsection{Devices under test}

To improve the understanding of modules operating under a series of different conditions, 2 devices were examined at test beam. The first device (LS4) was a barrel short strip sensor connected to a barrel hybrid populated with $10 \mathrm{ABC} 130$ (130 nm prototype) chips [4]. The sensor used was an ATLAS012 $300 \mu \mathrm{m}$ thick n-in-p micro-strip sensor developed by the ATLAS ITk Strip Sensor collaboration and produced by Hamamatsu Photonics [5]. In order to compare results for long and short strips under the same conditions, a long-strip module was approximated by connecting adjacent short-strip columns with wirebonds to form long $(4.8 \mathrm{~cm})$ strips. However, sections of the module were connected to only one column to allow direct comparisons between the two lengths on the same sensor connected to the same front-end ASIC (Fig. 1). In addition, a second device (DAQload10) consisting of a partially populated hybrid with three ABC130s and one ATLAS12 minisensor was assembled. This assembly allowed for rapid testing without using large number of ABC130s or full size sensors (Fig. 1).

\subsection{Test beam setup}

For the DESY test beams an electron beam energy of $4.4 \mathrm{GeV}$ was used, with tracking performed using the EUDET-style telescopes, which consist of six MIMOSA26 pixel sensors with a pitch of $18.4 \mu \mathrm{m}$ [6]. An additional pixel layer with an FEi4 [7] read-out was used to improve the timing of the telescope, allowing individual tracks to be matched to hits on the strip module under test. Tracks were reconstructed using the General Broken Lines algorithm [8], resulting in a pointing resolution of $2 \mu \mathrm{m}$ [6]. The strip modules were mounted between the third and fourth telescope planes. All devices were read out using the current test hardware (ATLYS FPGA development board) and software (ITSDAQ), integrated with the telescope data acquisition software (EUDAQ) [9]. The beam was incident on the surface of the device, with the size of the beam spot chosen to be $\sim 1 \mathrm{~cm}^{2}$, allowing for hits on strips wire-bonded to an individual $\mathrm{ABC} 130$ at a time. For each strip device, threshold scans were performed with a minimum of 200,000 events taken for each threshold setting ( $\sim \mathrm{k}$ events/strip). The scans were repeated for each sensor bias voltage being studied, and then for the different positions on the sensors.

\section{Results}

\subsection{Lab results}

The module LS4 was fully characterised in the lab for noise and gain by injecting a known amount of charge in the chip and performing a threshold scan. Shown in Fig. 2 are the data for both long and short strips whilst operated at $400 \mathrm{~V}$, where $V_{D E P}=370 \mathrm{~V}$. The increase in noise between long and short strips due to the difference in strip capacitance is in agreement with predictions from the ASIC design simulations [1]. 

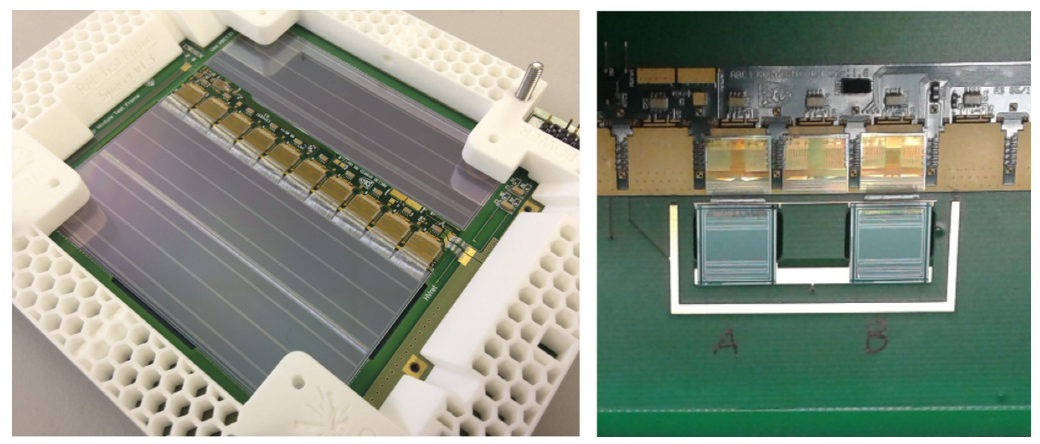

Fig. 1. Left: a fully assembled ABC130 Long-Strip Barrel Module (LS4); Right: a DAQload (DAQload10).
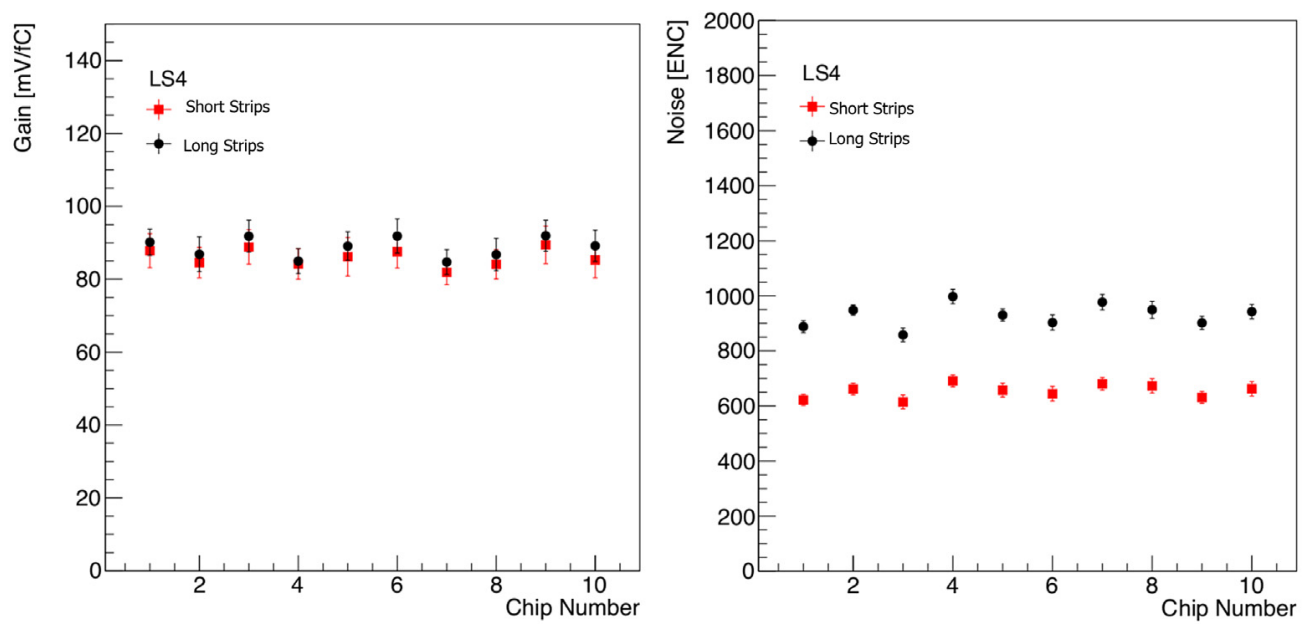

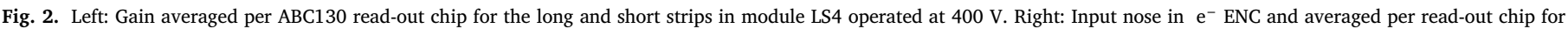
long-strip module utilising ABC130 chips, where the long strips are read out in Stream 1 and short strips in Stream 0.

\subsection{Module test beam results}

In all test beam measurements, threshold scans were performed and the tracks reconstructed with telescope data were used to determine the efficiency at each threshold. The efficiency is defined as the fraction of events in which a cluster is recorded whose centre is within $200 \mu \mathrm{m}$ from the track position as it passes the device under test. The threshold scans can be used to infer the distribution of the collected charge, as the difference between two points corresponds to the fraction of electrons producing a signal between those two threshold values. The threshold scans are then fit to a skewed error function, allowing for a determination of the most probable value (MPV) for the collected charge. The efficiency curves were evaluated at the DESY test beam for the long-strip and short-strip regions of the LS4 module at two different bias voltages, shown in Fig. 3. The differences in the curves arise from the module being operated in undepleted and over-depleted modes, where $V_{D E P}=370 \mathrm{~V}$. The noise occupancy as a function of the threshold is fit with an error function; the shape describes the distribution well with a minimal non-Gaussian tail in the noise spectrum [3]. The signalto-noise ratio yields values of 30 to 35 for a sensor bias voltage of $400 \mathrm{~V}[1]$.

The excellent pointing resolution from the telescope allows for investigation of the behaviour within and between the strips. Fig. 4 shows the hit occupancy as a function of the distance a track passes from the centre of a strip for a wide range of thresholds for DAQload10.

The curves show a flat efficiency in the central region of the strips, with a drop in the efficiency near the strip edges which is attributable to the effect of charge sharing between strips. Charge sharing can also be seen by looking at the average cluster size at relatively low thresholds (necessary for binary read-out systems), shown in Fig. 4. The likelihood of two-strip clusters increases for electrons passing in between two strips, where charge sharing is the highest.

\section{Conclusions}

An ATLAS ITk Strip barrel module was fully evaluated at a test beam for electrical performance. The efficiency is greater than the ATLAS specification of $99 \%$ for thresholds of up to $2.5 \mathrm{fC}$ at a bias voltage of $400 \mathrm{~V}$. The signal-to-noise ratio yields values of 30 to 35 . In addition, a flat efficiency in the central region of the strips with a width consistent with the strip pitch is measured. The results are as expected for a nonirradiated module and are well in agreement with the requirements for the ITk. Further test beams with irradiated parts are necessary to validate the modules and their components for end of life performance at the HL-LHC.

\section{Acknowledgements}

The research was supported and financed in part by Canada Foundation for Innovation, Canada, the National Science and Engineering Research Council (NSERC) of Canada under the Research and Technology Instrumentation (RTI) grant SAPEQ-2016-00015; the National Key Program for S\&T Research and Development (Grant No. 2016YFA0400101) of China and CAS-Helmholtz Joint Research Group, China; the Ministry 

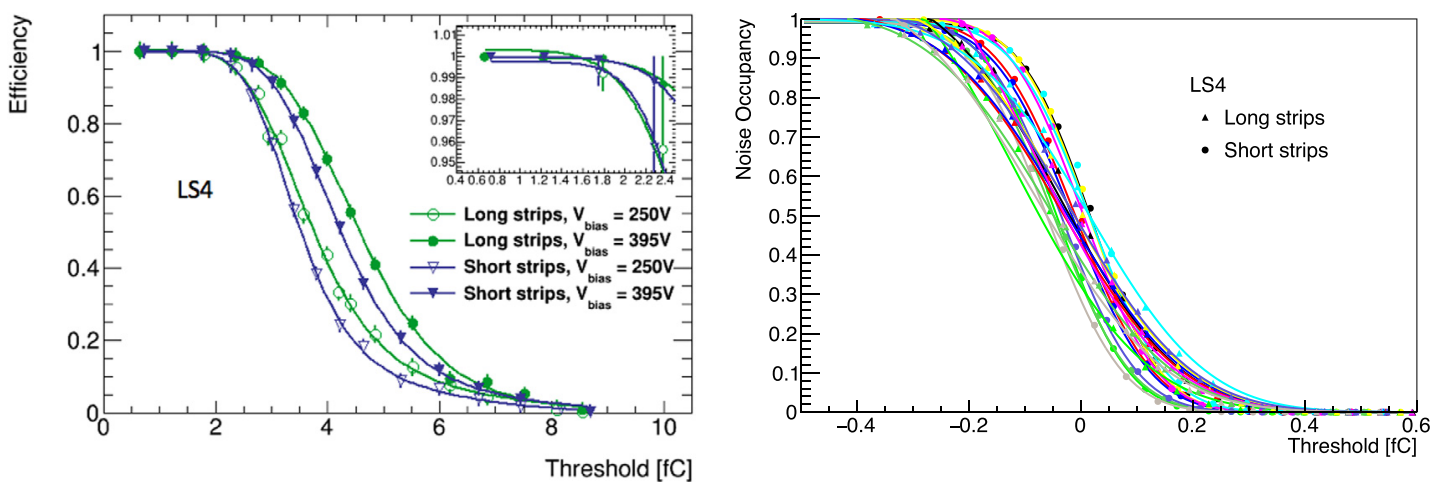

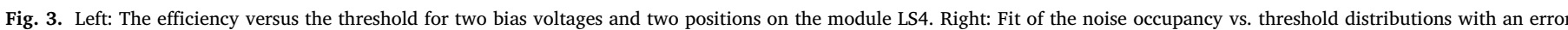
function (ERFC).
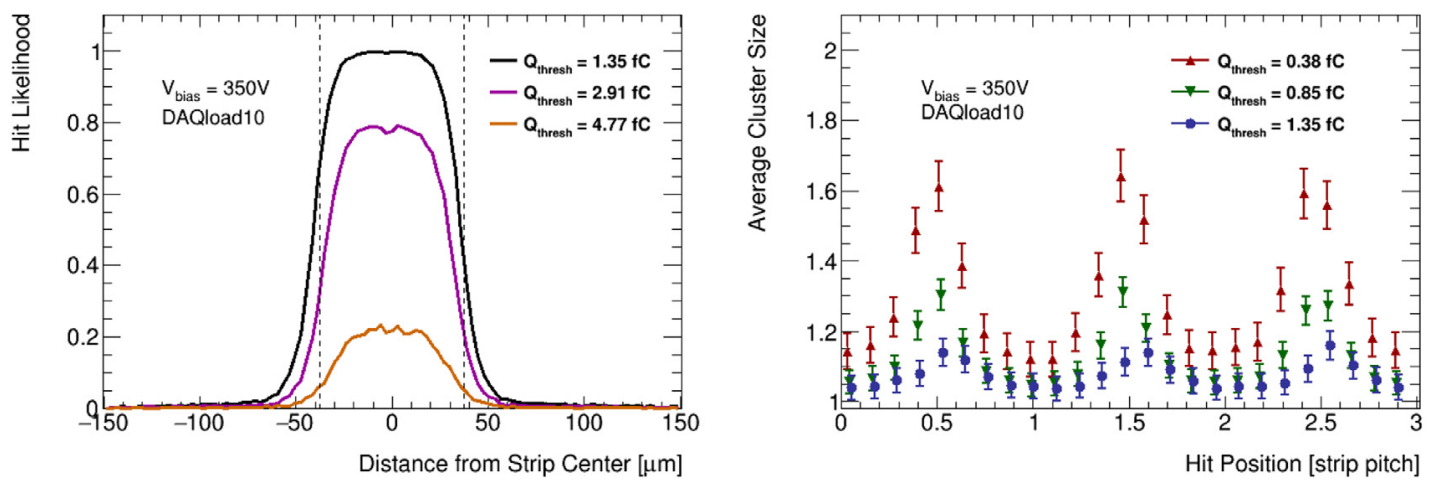

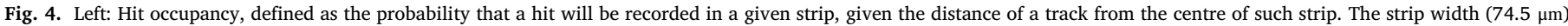

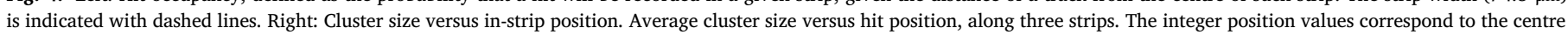
of the strips.

of Education, Youth and Sports of the Czech Republic coming from the project LM2015058-Research infrastructure for experiments at CERN; the DST/NRF in South Africa; the Spanish Ministry of Economy and Competitiveness through the Particle Physics National Program, Spain, ref. FPA2015-65652-C4-4-R (MINECO/FEDER, UE), and co-financed with FEDER funds, Spain; the UK's Science and Technology Facilities Council, UK; USA Department of Energy, USA, Grant DE-SC0010107; and the European Union's Horizon 2020 Research and Innovation programme, Spain, under Grant Agreement no. 654168. The measurements leading to these results have been performed at the Test Beam Facility at DESY Hamburg (Germany), a member of the Helmholtz Association (HGF).

\section{References}

[1] The ATLAS Collaboration, Technical Design Report for the ATLAS Inner Tracker Strip Detector, Technical Report CERN-LHCC-2017-005. ATLAS-TDR-025, CERN, Geneva, 04, 2017.
[2] The ATLAS Collaboration, The ATLAS experiment at the CERN large hadron collider, JINST 3 (2008) S08003.

[3] S. Diez, et al., A double-sided, shield-less stave prototype for the ATLAS Upgrade strip tracker for the High Luminosity LHC, J. Inst. 9 (2014).

[4] C. Argos, et al., The ATLAS ITk strip detector. Status of R\&D, Nucl. Instrum. Methods Phys. Res. A845 (2017).

[5] B. Hommels, et al., Detailed studies of full-size ATLAS12 sensors, Nucl. Instrum. Methods Phys. Res. A831 (2016).

[6] H. Jansen, et al., Performance of the EUDET-type beam telescopes, EPJ Technol. Instrum. (2016).

[7] M. Garcia-Sciveres, et al., The FE-I4 pixel readout integrated circuit, Nucl. Instrum. Methods A636 (2011).

[8] C. Kleinwort, et al., General Broken Lines as advanced track fitting method, Nucl. Instrum. Methods Phys. Res. A673 (2012).

[9] H. Perrey, et al., EUDAQ and EUTelescope software frameworks for test beam data acquisition and analysis, PoS 353 (2014). 